\title{
DIURNAL VARIATION OF THE OH CONCENTRATION IN AMBIENT AIR
}

\section{B. Shirinzadeh}

The Harrison M. Randall Laboratory of Physics, University of Michigan, Ann Arbor, Michigan 48109 Charles C. Wang*

Research Staff, Ford Motor Company, Dearborn, MI48121

$$
\text { D. Q. Deng }
$$

Department of Electrical Engineering, Wayna State University, Detroit, Michigan 48202

Abstract. $\mathrm{OH}$ concentrations in ambient air outside Ford Laboratory were measured in the months of July and August 1986 using the fluorescence technique at low pressures. The peak $\mathrm{OH}$ concentration was observed to be about 3 $x 10^{6}$ molecules $/ \mathrm{cm}^{3}$; the daytime average $\mathrm{OH}$ concentration was found to be in the range of middle $10^{5}$ molecules $/ \mathrm{cm}^{3}$, and the nighttime average below the detection limit of $0.9 \times 10^{5}$ molecules $/ \mathrm{cm}^{3}$. It is demonstrated that use of moving average may create the appearance of oscillatory behavior in the diurnal variations of the deduced $\mathrm{OH}$ concentration.

The hydroxyl radical (OH) plays a pivotal role in the transformation of trace species in the atmosphere, and has figured prominently in various photochemical models designed to gain insight into the chemistry of the atmosphere. Attempts have been numerous to make direct measurements of the $\mathrm{OH}$ concentration in ambient air. However, because of the high reactivity and low concentration of $\mathrm{OH}$, most of the techniques employed to date have been either inadequate in sensitivity, or susceptible to interference effects associated with laser-induced generation of $\mathrm{OH}$, or both; and definitive results of $\mathrm{OH}$ measurements do not appear to have been available.

Most recently, Hard et al [1986] reported $\mathrm{OH}$ measurements using the fluorescence technique at low pressures. They reported a detection limit (at one standard deviation) of $2 \times 10^{5}$ molecules $/ \mathrm{cm}^{3}$ with one hour averaging, and noted the appearance at night of several peaks in the $\mathrm{OH}$ concentration at a level of nearly $1 \times 10^{6}$ molecules $/ \mathrm{cm}^{3}$. The purpose of this letter is to report our $\mathrm{OH}$ measurements which were also conducted with the fluorescence technique at low pressures, but with a much reduced energy density for excitation. (For nighttime monitoring, it is about a factor of one thousand lower than that used in the previous measurements.) With the intensity level used for excitation in our measurements, a daytime peak $\mathrm{OH}$ concentration as high as $1.8 \times 10^{6}$ molecules $/ \mathrm{cm}^{3}$ was of ten observed, but no $\mathrm{OH}$ concentration above the detection limit of $0.9 \times 10^{5}$ molecules $/ \mathrm{cm}^{3}$ was noted at night. We have also found that the apparent oscillatory behavior in the diurnal variation of the OH signal observed by these *To whom correspondence regarding this paper should be addressed.

Copyright 1987 by the American Geophysical Union. Paper number 6L6437. $0094-8276 / 87 / 006 \mathrm{~L}-6437 \$ 03.00$ authors may have been an artifact resulting from data averaging.

The measurements to be reported in this letter were performed outside Ford Laboratory in Dearborn, Michigan, with the output from a tunable dye laser pumped by a doubled Nd-YA1G laser 'Bakalyar et al, 1984]. This output in the ultraviolet was tuned to be in resonance with the Q1(2) transition of $\mathrm{OH}$ near $282.1 \mathrm{~nm}$. A small percent of the output beam was split off from the main beam and directed into a water vapor discharge, where copious amounts of $\mathrm{OH}$ fluorescence excited by the output beam helped ensure the spectral position of the radiation. The main part of the beam was expanded, traversed a light-shielded stainless steel cell, and was then detected. The cell windows were located some $60 \mathrm{~cm}$ away from the center of the fluorescence chamber, thus contributing little to the observed background fluorescence. The fluorescence signal excited in the chamber was collected with a 3-inch lens operated in an $f / 3$ configuration, processed through appropriate spectral filtering, and detected by a highgain photomultiplier tube. The signal from the photomultiplier was time-gated and processed by a photon counter. An HP-16 computer provided data storage and manipulation.

For our $\mathrm{OH}$ measurements, the laser was operated at a repetition rate of 30 pulses per second, and was tuned on and off the $\mathrm{OH}$ transition every 50 pulses. The output pulse near $282.1 \mathrm{~nm}$ typlcally measured $0.5 \mathrm{~mJ}$ in energy, $10 \mathrm{nsec}$ in duration, and $0.1 \mathrm{~cm}^{-1}$ in linewidth. The effective beam cross section within the fluorescence cell was about $2 \mathrm{~cm}^{2}$, giving an energy density for excitation of 0.25 $\mathrm{mJ} / \mathrm{cm}^{2}$. Ambient air was directed to flow into the fluorescence chamber through an orifice 0.3 $\mathrm{im}$ in diameter [Shirinzadeh et al, to be published, 1987]. At a flow rate of about 0.8 11 ter/min, the pressure of the chamber was maintained at about 3 torr. The concentration of ozone and water were measured immediately before the orifice with a DASIBI ozonemeter and a hygrometer respectively. As before [Bakalyar et al, 1984], OH concentrations were deduced from the observed fluorescence signal by normalizing it to the Raman signal due to molecular nitrogen observed concurrently, and a rotational distribution thermalized at the ambient temper.3ture was assumed for the ambient $\mathrm{OH}$.

In order to quantify the extent of ozone interference in the observed OH signal, measurements were made on ozone-enriched ambient air before and after $\mathrm{OH}$ monitoring to determine 

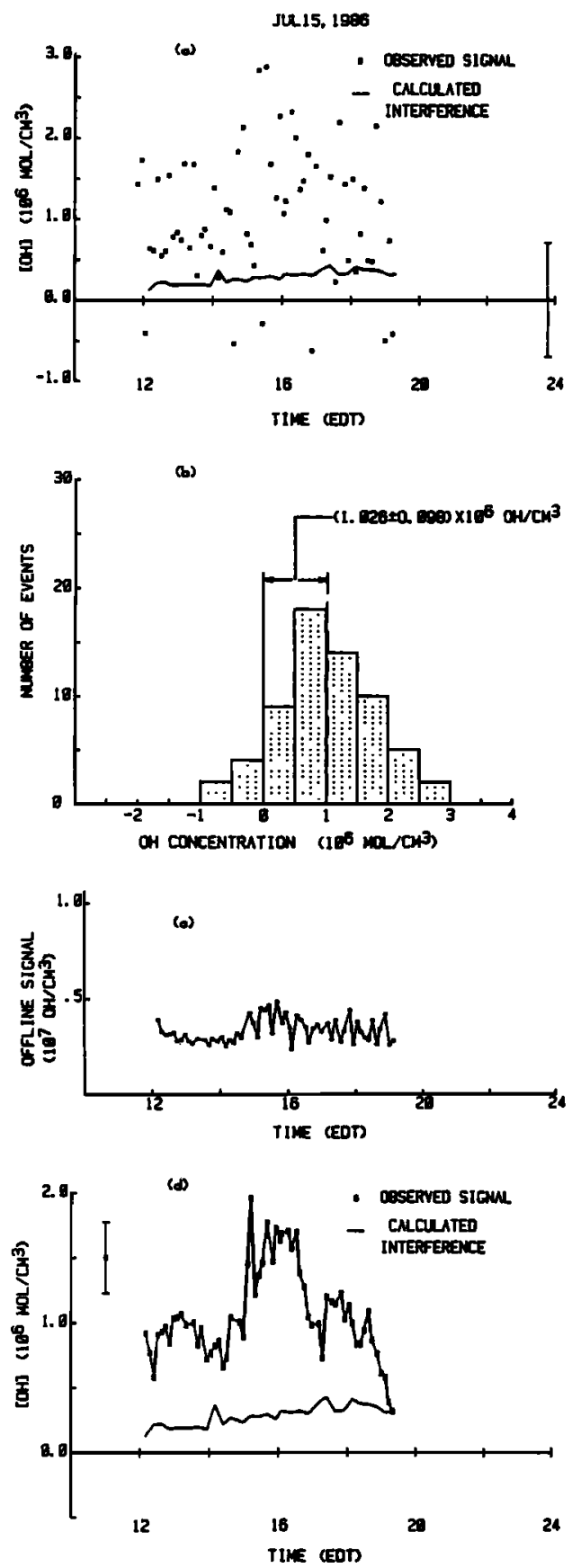

Fig. 1 Results of OH measurements in July 15 , 1986: (a) Deduced OH concencrations, with each data point representing measurements of six minutes and the error bar representing the statistical uncertainty associated with the measurements; (b) Histogram for the distribution of the deduced OH concentrations; (c) Deduced values for the background fluorescence with six minute averaging; and (d) Same results as in (a) presented with a 40-minute (six point) moving average.

the level of ozone interference due to given concentrations of ozone and water. With this information, the level of ozone interference in the observed oH signal could then be subtracteo according to the observed ozove and water level
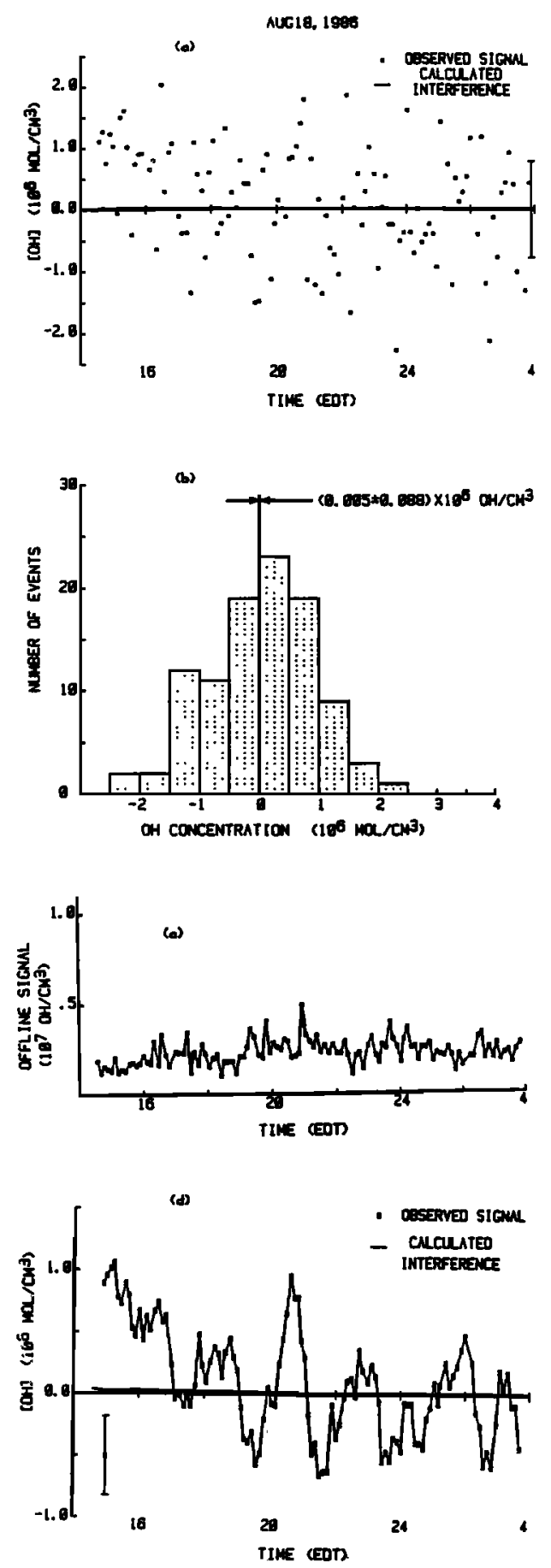

Fig. 2. Results of $\mathrm{OH}$ measurements in August 18, 1986: (a) Deduced OH concentrations; (b) Histogram for the distribution of the deduced $\mathrm{OH}$ concentrations; (c) Deduced values for the background fluorescence with six minute averaging; and (d) Same results as in (a) presented with a 40-minute (six point) moving average.

[Shirinzadeh et al, to be published, 1987]. For nighttime measurements concern for possible interference due to laser-induced dissociation of species other than ozone led us to reduce the exciting energy per pulse by a factor of two and to increase the beam cross section by another 
TABLE I.

Sumaary of Results of OH Measurements in Dearborn, Michigan, in the Months of July and August, 1986

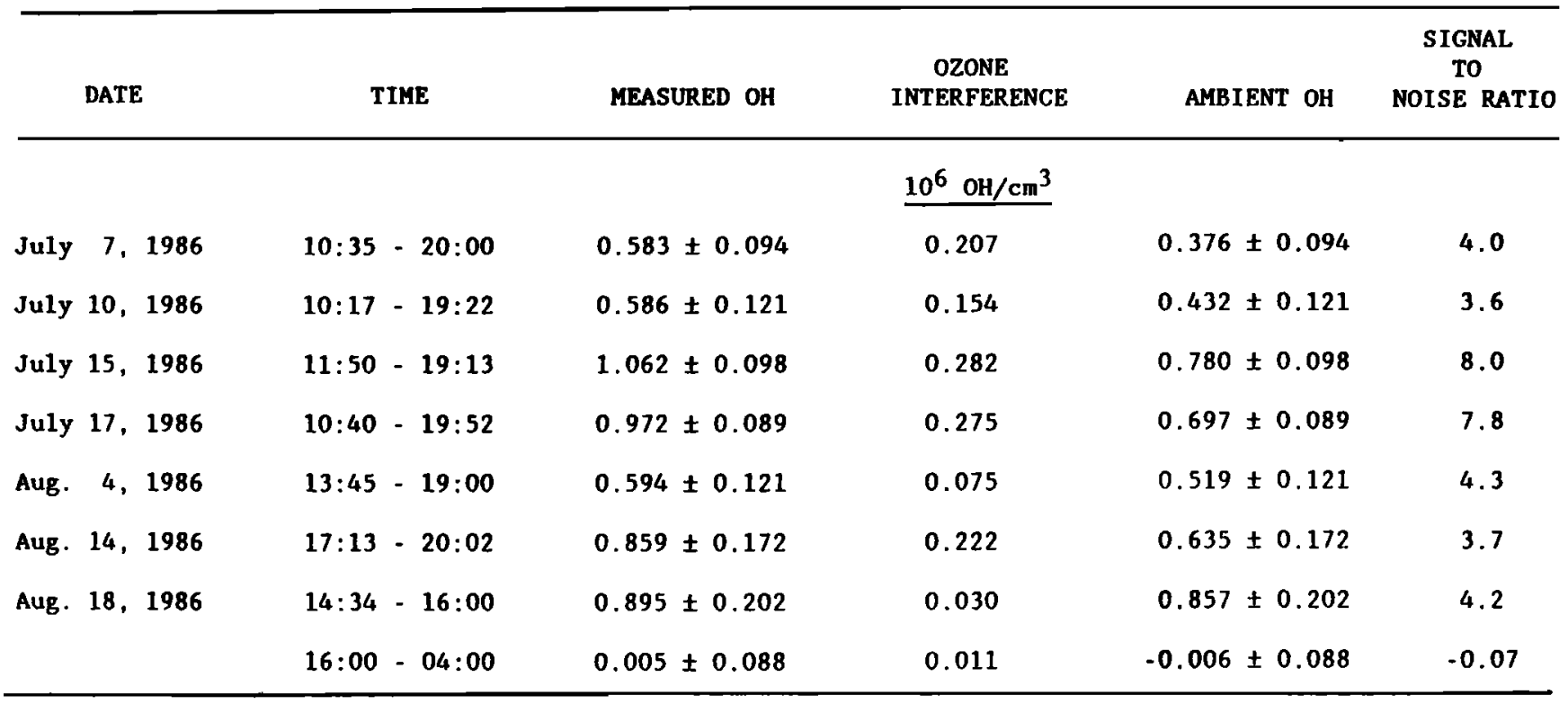

factor of 2.5 , thus reducing the exciting energy density to $0.05 \mathrm{~mJ} / \mathrm{cm}^{2}$.

At the flow rate used in our measurements, the residence time of the sampled air inside the fluorescence chamber was estimated to be about one second. In an effort to estimate the loss of OH through the sampling process, we have generated high levels of $\mathrm{OH}$ in ambient air using a heat gun, and have varied the residence time through changes in the volume of the fluorescence chamber, the size of the orifice and the flow rate. No apparent change in the $\mathrm{OH}$ signal was observed when the residence time was either decreased or increased by an order of magnitude. These and other results indicate that the loss of OH through the sampling process is probably negligible.

In Fig. 1a, the results of $\mathrm{OH}$ measurements are shown for the afternoon of July 15, 1986. The corresponding level of ozone interference as determined from the ozone and water level measured concurrently is also shown. Here each data point represents an $\mathrm{OH}$ value deduced from 10,000 laser shots over a period of approximately six minutes. These data appear to show a peak in the $\mathrm{OH}$ concentration around 15:30 EDT (Eastern Daylight Time), with a peak value of about $3 \mathrm{x}$. $10^{6} \mathrm{oH} / \mathrm{cm}^{3}$. Statistical analysis (Fig. Ib) also shows that these results are distributed approximately normally with a mean value of 1.06 $x 10^{6} \mathrm{oH} / \mathrm{cm}^{3}$ and a standard deviation of the mean of $0.098 \times 10^{6} \mathrm{OH} / \mathrm{cm}^{3}$. During these measurements, the fluorescence background was found to be essentially constant (Fig. 1c); and the shot noise associated with this background was found, according to photon statistics, to contribute to the mean value an uncertainty of $0.09 \times 10^{6} \mathrm{oH} / \mathrm{cm}^{3}$. It would thus seem prudent to conclude that this shot noise is the primary factor in determining the detection limit.

For the purpose of comparison, the results in Fig. la are replotted in $\mathrm{fig}$. 1d with a 40-minute (six point) moving average. The appearance of several peaks, as was also apparent in the results of Hard et al [1986], seems intriguing. We feel, however, that this is in part an artifact resulting from the use of moving average. This conclusion follows from the fact that moving average is analogous to a low pass filter which retains the low frequency components of the signal as well as those of the noise background, and that the latter may be order of magnitude larger than the former.

In Fig. 2, similar results obtained during the night of August 18 through August 19, 1986 are shown. The corresponding level of ozone interference was determined to be quite low, the fluorescence background was found to be at a comparable level, and the data polnts are also found to be distributed approximately normally. For the period between 16:00 EDT and 04:00 EDT, this distribution may be associated with a mean value of $0.005 \times 10^{6} \mathrm{OH} / \mathrm{cm}^{3}$ and a standard deviation of the mean value of $0.088 \times 10^{6}$ $\mathrm{OH} / \mathrm{cm}^{3}$.

We have made repeated observations during the summer of 1986, and the results are generally similar to those presented above. It is seen in Fig. 2 that the OH signal drops below the noise level at sunset. However, before the much reduced intensity was used for nighttime measurements, the $\mathrm{OH}$ signal was seen to rise again after sunset with predictable regularity. One possible source for this problem was the formation near sunset of other species which could be dissociated to form $\mathrm{OH}$ by the exciting laser beam. The presence of this unknown source of interference makes it extremely important to avoid use of any intensity-enhancing schemes such as White cells, and to excite the fluorescence of OH with the lowest possible energy density.

Table I summarizes the results of $\mathrm{OH}$ measurements conducted in the months of July and August 1986 in Dearborn, Michigan. The levels of 

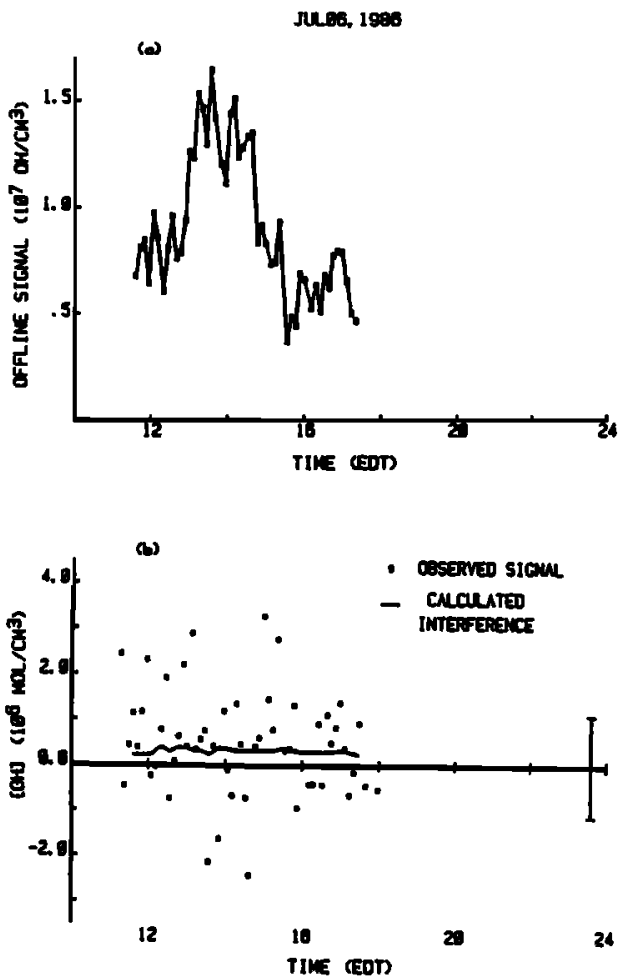

F1g. 3. (a) Background fluorescence deduced from OH measurements on July 6,1986 with six minute averaging; (b) the corresponding deduced $\mathrm{OH}$ concentration with 6 -minute averaging.

ozone interference, as determined from concurrent measurements of ozone and water concentrations and averaged over the perfods indicated are also given. It is seen that the ozone interference encountered in the measurements was usually at a level of low $10^{5} \mathrm{oH} / \mathrm{cm}^{3}$, becoming even lower when the energy density for excitation was reduced to $0.05 \mathrm{~mJ} / \mathrm{cm}^{2}$. The values of ambient $\mathrm{OH}$ concentration were obtained by subtracting the computed level of ozone interference from the values for $\mathrm{OH}$ concentration deduced from fluorescence measurements. These values are seen to cluster around the middle $10^{5} \mathrm{OH} / \mathrm{cm}^{3}$ with reasonable precision.

In the measurements presented above, the fluorescence background stays relatively constant throughout the course of a day; even though this background changes from day to day, it is usually in the range equivalent to low $10^{6} \mathrm{OH} / \mathrm{cm}^{3}$. This point is amply demonstrated by comparing the background levels observed in Fig. $1 \mathrm{c}$ and Fig. $2 c$, and by noting the standard deviation of the mean calculated for the measurements of July 15 and August 18. The fact that the mean value for the nighttime measurements on August 18 is zero within one standard deviation of the mean suggests very strongly that no systematic bias such as that due to slanting baseline exists in our measurements. This conclusion is also supported by other nighttime measurements in which the laser frequency was tuned to either side of the $\mathrm{OH}$ resonance, and no difference beyond the statistical uncertainty of the measurements was ever observed. There were occasions, however, when the background fluorescence became excessively high and fluctuating over a wide range (Fig. 3a), leading to wild excursions in the values for the $\mathrm{OH}$ concentration deduced from fluorescence measurements (Fig. $3 \mathrm{~b}$ ); it is possible this elevated background level was associated with pockets of severely polluted air.

Based on the results presented above, it seems highly desirable to conduct $O H$ monitoring with an energy density for excitation maintained at, say $0.05 \mathrm{~mJ} / \mathrm{cm}^{2}$. Through proper redesign of the collection optics, it should be possible to maintain the energy density for excitation at this level without reducing the energy per pulse, thus improving the rate of signal return. Although it was inadequate in sensitivity when used in the LIDAR configuration, our detection system has proved to be operationally reliable in past field measurements of $\mathrm{OH}$. With this change to fluorescence detection at low pressure, routine meaningful measurements of $\mathrm{OH}$ should now be possible.

This research was supported in part under a contract from the Department of Energy.

\section{References}

Bakalyar, D. M., L. I. Davis, C. Guo, J. V. James, S. Kakos, P. T. Morris, and C. C. Wang, Shot Noise Limited Detection of $\mathrm{OH}$ Using the Technique of Laser Induced Fluorescence, ApDl. Opt., 23, 4076, 1984

Hard, T. M., C. Y. Chan, A. A. Mehrabzadeh, W. H. Pan, and R. J. O'Brien, Diurnal Cycle of Tropospheric OH, Nature, 322, 617, 1986.

(Received November 24, 1986; accepted December 30, 1986.) 